Running head: Communicative hand movements in young infants

Hand movements in communicative and non-communicative situations in very young infants: a preliminary study

Somogyi, E*, Salomon, L. ** \& Fagard, J. *

*Laboratoire Psychologie de la Perception, Université Paris Descartes, Paris, France

**Hôpital Universitaire Necker-Enfants Malades, Paris, France

Corresponding author:

Eszter Somogyi*

Address : Université Paris Descartes, 45, rue des Saints-Pères, 75006 Paris, France

E-mail: eszter.somogyi@port.ac.uk

Phone: +44 7849363131 


\title{
Hand movements in communicative and non-communicative situations in very young infants: a preliminary study
}

\begin{abstract}
As a step towards understanding the developmental relationship between handedness and language lateralization, this longitudinal study investigated how infants $(\mathrm{N}=21)$ move their hands in non-communicative and communicative situations at two weeks and at three months of age. We looked at whether left-right asymmetry in hand movements and in duration of selftouch appeared across conditions and whether the direction of asymmetry depended on the communicative nature of the situation. We found that asymmetries appeared less consistently than suggested in literature and did not only depend on the communicative nature of the situation. Instead, hand activity and self-touch patterns depended on age, the presence of the mother, the degree of novelty of the situation and the presence of an object. Our results partly support previous studies that pointed out an early differentiation of communicative hand movements versus non-communicative ones in infants. It is in terms of the amount of global hand activity, rather than in those of the laterality of hand movements that this differentiation emerged in our study. At three months, infants moved their hands more in the communicative conditions than in the non-communicative conditions and this difference appeared as a tendency already at two weeks of age.
\end{abstract}

Key words: hand movements; asymmetry; communicative vs. non-communicative condition; mother vs. stranger; infant

\section{Introduction}

Handedness and hemispheric dominance for language are the two most studied forms of asymmetry and the relationships between the two have long been the focus of research, mainly for two reasons. The first is the high prevalence of left hemispheric dominance for both functional asymmetries, namely that $90 \%$ of right-handers are left-hemisphere dominant for language. The second is that, despite the fact that atypical hemispheric dominance for language in adults is more frequent in left-handers than in right-handers (Knecht, 2000), still $70 \%$ of left-handers show, like right-handers, left-hemisphere dominance for language. We therefore can seriously question the nature of the link between handedness and language lateralization (Fagard, 2013). One possible way to understand the nature of the relationship between handedness and language lateralization is to investigate and compare their early 
development. Signs of handedness appear prenatally, as we will see below, and handedness can be tested in infants prior to the emergence of spoken language. But signs of lateralization for language perception are also very precocious, and can be detected even at birth in preterm infants (Mahmoudzadeh et al., 2013). In addition, some indication of lateralization of language production can be observed before real words are emitted, for instance in the production of communicative gestures (Trevarthen, 1996; Capirci \& Volterra, 2008) or in mouth opening during babbling (Holowka \& Petitto, 2002). Although some studies have looked at handedness for gestures in older infants (Cochet, Centelles, Jover, Plachta, \& Vauclair, 2015; Esseily, Jacquet, \& Fagard, 2011), to our knowledge, few studies have examined hand use in communicative situations during the very first months of life. Therefore, the aim of the present study was to compare how infants move their hands in communicative and non-communicative situations at two weeks and at three months of age. Before presenting our research, we will first review existing literature on the early development of handedness, followed by an overview of studies of handedness for early communicative gestures. In this review, we will use the term gesture to refer to infants' hand movements observed in communicative situations.

\subsection{Early development of handedness}

Prenatal signs of postural asymmetries and lateralized hand movements have been reported by several studies using ultrasound recordings. In one study, the authors observed 282 fetuses repeatedly, starting from the $15^{\text {th }}$ week of gestation and reported that $80 \%$ of the fetuses sucked their right thumb more than the left (Hepper, Shahidullah, \& White, 1991). A prevalence of right-arm over left-arm movements has also been reported in a further study by McCartney and Hepper (1999) who observed 17 fetuses every three weeks from the $12^{\text {th }}$ to the $27^{\text {th }}$ week and by Kurjak et al. (2002) who observed 98 fetuses between the $6^{\text {th }}$ and $12^{\text {th }}$ week of gestation.

However, these observations have not always been replicated. For instance, in one study concerning 10 fetuses seen every four weeks between the $10^{\text {th }}$ and $38^{\text {th }}$ week (de Vries et al., 2001), there were very few instances of thumb sucking and these were not performed more often with the right hand (RH) than the left hand (LH). In a different study, head position of the fetus was found to become increasingly lateralized from 30 weeks of gestational age onward with an evident right-sided preference by 36 weeks, but hand movements toward the face were not lateralized (Ververs, de Vries, van Geijn, \& Hopkins, 1994). Similarly, in still another study of 27 fetuses seen once between the $19^{\text {th }}$ and $35^{\text {th }}$ week of pregnancy, no significant difference has been observed between the occurrences of hand- 
to-mouth movements with the LH or the RH (Myowa-Yamakoshi \& Takeshita, 2006). Thus, whether signs of handedness appear before birth is still an open question.

At birth, a few studies indicate more right- than left-arm movements in infants, but this asymmetry seems to originate from postural head asymmetry. In one study (involving 12 fullterm newborns), the infants showing a tendency to turn their head toward the right (asymmetric tonic neck reflex, ATNR) touched their mouth with their RH more often than with their LH, whereas no general difference was found between $\mathrm{RH}$ and the $\mathrm{LH}$ for other movements (Hopkins, Lems, Janssen, \& Butterworth, 1987). In an earlier study, no evidence of laterality was found in newborns' spontaneous movements, that is without a stimulus, whereas the introduction of a visual stimulus induced more RH than LH movements directed toward the target (Rader \& Stern, 1982). In one longitudinal study, it was found that a stable tendency to orient the head toward one side during the first weeks predicts rather well handedness for spontaneous movements at 3-8 weeks, hand movements in front of an object at 12 weeks, and handedness for reaching at 16 to 74 weeks (Michel \& Harkins (1986), who observed 10 infants with right-side ATNR and 10 infants with left-side ATNR at birth; see also: von Hofsten, 1982). These studies stress the role of postural head asymmetry in manual asymmetry before reaching: very young infants tend to turn the head toward one side (most often the right) and they move more the hand they see (see, for instance: Van Der Meer, 1997). Note that, like Rader and Stern (1982), Michel and Harkins (1986) did not find any difference in the frequency of left- versus right hand movements when the infant's head was in the midline position in the absence of object at 12 weeks, whereas in pre-reaching movements, that is in the presence of a visual stimulus, there were more RH- than LH movements directed toward the target. However, this result is not in accordance with a more recent kinematic study where 13 infants were followed from eight weeks to reach onset: in this study there was no strong evidence of left-right difference in pre-reaching movements in any of the variables studied, including movement frequency and whether a toy was present or not (Lynch, Lee, Bhat, \& Galloway, 2008).

Thus, some studies indicate that, at least with an object presented in the midline, prereaching infants move their right arm more than the left, when their posture is symmetrical. Once reaching and grasping emerge, the frequency of infants with $\mathrm{RH}$ preference largely exceeds that of infants with LH preference: this right-handedness seen at the population level is accompanied by large individual fluctuations occurring until late into the second year (Corbetta \& Bojczyk, 2002; Fagard \& Dahmen, 2004; Fagard, Margules, Lopez, Granjon, \& 
Huet, 2017; Kotwica, Ferre, \& Michel, 2008; Michel, Babik, Sheu, \& Campbell, 2014; Michel, Tyler, Ferre, \& Sheu, 2006; Nelson, Campbell, \& Michel, 2013).

\subsection{Early lateralization of hand movements in communicative situations / gestures}

Hand movements of infants engaged in proto-conversation with their mother have been proposed to be different from movements made when the infant is looking at an object. From the analysis of video recordings made during infants' first year, Trevarthen (1996) reached the conclusion that utterances of cooing or 'pre-speech' were often accompanied with RH movements. In particular, this author counted the number of movements made by six 4- to 24week-old infants in interaction with their mother or with a stranger. He compared the frequency of RH and LH movements for gesturing (raising the hand) and self-touching while in communication. No statistics are given but the author observed that most hand raising movements toward the mother were made with the RH already at four weeks. There were many fluctuations within infants across sessions, but it is interesting to note that all six infants raised more often their RH than their LH toward their mother already at the first session, and that five out of the six infants raised more often their RH if one considers the mean across all sessions. In contrast, self-touching movements were most often made using the LH, and all six infants produced more LH movements for self-touching than for hand raising. Self-touch with the LH could be a self-regulatory response facilitating attentional focus, as shown in adults when responding to emotional or cognitive load (e.g. Mueller, Martin \& Grunwald, 2019). Another interesting point is that when the conversation was with a stranger, the infants were more active with their LH than with their RH, which, Trevarthen (1996) suggested, may be associated with novelty or potential distress.

Whilst Trevarthen (1996) did not systematically compare self-touch across sessions (only hand activity), Reissland, Aydin, Francis \& Exley (2015) provide further evidence in fetuses to suggest that self-touch with the LH may be associated with potential distress. They observed that self-touch with the LH was positively related to maternally reported stress levels. It is therefore feasible that LH activity and self-touch with the LH are not incidental or equivalent to the hand being in the air or away from the body, but instead a self-regulatory response to novelty or mild stress. Furthermore, these occurrences of lateralized self-touch appear to be distinct from spontaneous self-touch. A recent study that examined the development of spontaneous self-touch between 3 and 13 weeks of age did not find lateral differences between hands (DiMercurio, Connell, Clark \& Corbetta, 2018).Pointing, considered as the earliest true communicative movement, and as a form of pre-language (Capirci \& Volterra, 2008), has been the focus of several studies of handedness. Pointing is 
defined as the extension of the index finger while the other fingers remain retracted, combined with arm extension. Mature indisputable pointing is not frequent before the beginning of the second year of life, but there is some debate about the meaning of very young infants' index finger extension in the absence of arm extension (Blake, O'Rourke, \& Borzellino, 1994; Trevarthen, 1996). In one study with 16 dyads observed monthly up to seven times between 3 to 12 months (Hannan, 1987) the authors observed many instances of 'pointing' already at 3 months of age. Significantly more pointing occurred when the infant faced his/her mother in the absence of an object ('mother-alone') than when the mother shook a toy in front of $\mathrm{him} / \mathrm{h}$ r. In addition, there was a tendency for more pointing to occur with the RH in the mother-toy condition, and significantly more pointing with the LH in the mother-alone condition.

We can note that only few studies have looked at the early lateralization of hand movements in communicative situations. These seem to indicate that asymmetries in hand raising movements and in pre-pointing gestures appear already during the first months of life when the infant is in a communicative situation. Furthermore, the direction of asymmetries in hand movements appear to differ depending on the person that the infant is facing (mother or stranger) and on what the mother is doing (communicating only or shaking an object). We shall now review studies that directly compared how communicative hand movements and manipulative actions develop during early childhood.

\subsection{Comparison of hand preference for communicative and non-communicative movements}

Studies comparing older infants' hand preference for grasping and hand preference for communicative and symbolic gestures will not be detailed here as the present research focused on infants younger than 4 months of age. Most of these studies suggest however that manual asymmetries toward right-handedness are larger for infants' communicative and symbolic gestures than for manipulative actions (Bates \& Dick, 2002; Bates, O'Connell, Vaid, Sledge, \& Oakes, 1986). For instance, in a study of 4-, 8- and 12-month old infants (12 infants for each age group), the authors observed that index finger extension, also referred to as 'pre-pointing', was evenly split between both hands at 4- and 8 months whereas pre-points and points were right-handed at 12 months (Blake et al., 1994). Interestingly, reaching for something out of reach (communicative movement) was right-handed at 8 and 12 months, whereas reaching for something within reach occurred equally often with either hand at 4 and 8 months, and became more right-handed only at 12 months. These and other results on older children (Cochet \& Vauclair, 2010; Esseily et al., 2011; Jacquet, Esseily, Rider, \& Fagard, 
2012; Vauclair \& Imbault, 2009) seem to indicate that the development of manual asymmetries follows a different trajectory for object manipulation and communication (see Cochet et al., 2015, for a review).

Yet, a few studies point to a relationship between the emergence of hand preference for grasping and the development of language. For instance, in a longitudinal study, Ramsay (1984) found that the onset of unimanual handedness in infants co-occurs with the emergence of babbling and duplicated syllables. The author concluded that the ability to duplicate syllables and the emergence of handedness both reflect developmental change in hemispheric specialization. The same observation was made by these authors for bimanual handedness (Ramsay, 1980). A more recent study showing that the stability of right-handedness at seven months is correlated with a higher level of vocabulary at 24 months (Michel, Babik, Nelson, Campbell, \& Marcinowski, 2013) offers further evidence of a possible relationship between handedness and early language development (see also: Cochet, Jover, \& Vauclair, 2011). Finally, hand preference for pointing has also been shown to be correlated with the development of language in 12- to 30-month-olds (Vauclair \& Cochet, 2013), as well as in 14-month-old children (Esseily et al., 2011).

In summary, existing developmental studies with older infants and toddlers indicate that while the onset of handedness and language development may be linked, gesture lateralization is not a mere consequence of object-related handedness. Humans may have a specialized area for communicative gestures and language in the left cerebral hemisphere that could be independent of the system controlling the purely motor functions of hand use.

\subsection{The present study}

As we could see, the above studies mostly involved fetuses, older infants and toddlers, therefore the investigation of hand movements during the very first months of life is needed in order to learn how early in development these asymmetries emerge. With our present study we wished to fill this gap by analyzing and comparing infants' hand activity and duration of self-touch in communicative and non-communicative situations, at two weeks and at three months of age. In the communicative situation, infants' hand movements were recorded while the mother or a female experimenter talked to the two-week- or the three-month-old infant, with her face within the infant's sight, using motherese. In the non-communicative situation, the mother or the same female experimenter gently shook a rattle without talking this time, but again with her face within the newborn's or infant's sight. Note that although the mother's or the female experimenter's face was visible in both conditions and therefore both situations were potentially communicative, in this study we will refer to our conditions as 
'communicative' and 'non-communicative' because the infant was addressed in motherese in the former condition whereas in the latter, the mother and the experimenter were asked to be silent. A fifth, baseline condition was also recorded, where no stimulation was provided. Infants' hand movements were analyzed in order to compare the quantity of hand movements and the duration of self-touch across the five conditions. We added a female experimenter other than the mother in order to investigate the effect of familiarity. Indeed, all studies of infants' lateralized hand movements during communication with a person have been restricted to communication with the mother. It is known that infants show a preference towards the voice of the mother as compared to that of another female (Decasper \& Fifer, 1980). Thus, we wished to investigate whether such an effect would also be present when facing a female experimenter in our study.

Our four hypotheses were the following. First, we expected that at baseline, when infants are positioned in a symmetrical posture and no stimulation is provided, hand activity and duration of self-touch would not be lateralized at two weeks or at three months of age (Michel \& Harkins, 1986; Rader \& Stern, 1982; DiMercurio, et al., 2018).

Second, we hypothesized that three-month-old infants would produce different patterns of hand activity and self-touch in the communicative situation with their mother compared to the communicative situation with the stranger. Specifically, we expected that they would produce more RH (vs LH) activity when facing their mother, but show the opposite, more LH (vs RH) activity when facing the stranger, as a response to the novelty of the situation (Trevarthen, 1996). When facing the mother, we also expected infants to touch their own body for longer durations with the LH (vs RH), as a self-regulatory response (Trevarthen, 1996; Mueller, 2019). Finally, we expected infants to produce more self-touch with LH when facing the stranger (vs the mother) as a potential response to the mild stress involved (Reissland et al., 2015).

Third, we expected that in the non-communicative situation where a rattle is presented at midline, three-month-old infants would produce pre-reaching ${ }^{1}$ movements, with more RHthan LH movements directed toward the target (Michel \& Harkins, 1986; Rader \& Stern, 1982).

We therefore expected a $\mathrm{RH}$ preference for hand movements in the communicative situations as well as for non-communicative pre-reaching actions (e.g.: Bates et al., 1986;

\footnotetext{
${ }^{1}$ We did not have the possibility to differentiate movements towards the object from other movements. Thus, by 'pre-reaching movements', we mean 'movements in the presence of an object' made by infants before they are able to touch and grasp objects.
} 
Blake et al., 1994; Kimura, 1973). Our fourth hypothesis was that, since the lateralization of hand movements in communicative situations seems to develop independently from objectrelated handedness (Cochet et al., 2011; Jacquet et al., 2012), different patterns of hand activity and self-touch would emerge when comparing hand movements in communicativeand non-communicative situations to baseline. Since no studies to date have compared hand movements in these two situations, we did not formulate specific expectations as to the direction of the differences we would observe.

\section{Method}

\subsection{Participants}

The hand movements of 21 infants were recorded at two weeks $\left(2 \mathrm{w}: M_{\text {age }}=11\right.$ days, $S D=8$ days, 5 boys $)$ and at three months of age ( $3 \mathrm{~m}: M_{\text {age }}=93$ days, $S D=7$ days). Families were recruited in the framework of a greater longitudinal research project on the origins of manual lateralization and hemispheric asymmetries for language processing. Parents were informed about the study at their pregnancy counselling in a mainstream public hospital in Paris, France. Parents who were interested in participating received a detailed information sheet. Tear-off forms were appended to the sheet for parents to return their contact details, allowing us to call them in order to provide further information. Those who agreed to participate gave their signed informed consent on the occasion of the first observation. A total of 32 healthy, full-term newborn infants were recruited for the present study. The data of 21 infants were retained for the final sample. The remaining 11 infants were excluded due to unavailability of the mother following birth $(n=5)$, birth complications $(n=2)$ and fussiness of the infant making the observation at one of the two time points impossible $(n=4)$.

\subsection{Procedure}

Two-week-old infants were tested either at the hospital $(n=7)$ or in their home $(n=14)$. At three months, all infants were tested in their homes. The observation was timed just after the infants' feeding time and started when the infant was in a state of calm rest. Infants were tested in a quiet, calm room. In order to avoid movement biases towards brighter surfaces, infants were positioned with their back to any source of light (window or lamp). Care was also taken to avoid any possible biases towards interfering sound sources. Any clothing that could have inhibited free movements of the arms was removed. Infants were tested without a pacifier. Only the mother and the experimenter were present during the observation. Once the infant was ready, he or she was placed in a semi-supine position in a baby seat, strapped in at the waist, with the arms free to move. The camera was placed behind and above the infant, at 
$1,5 \mathrm{~m}$ from the infants' head, in order to have a complete view of both hands and to avoid obstruction by the adult's head.

We recorded infants' hand movements across the following five conditions, each lasting 40 seconds:

1. Baseline condition: the infant's spontaneous hand movements were recorded

2. Communicative-Mother: the mother talked to the child

3. Communicative-Experimenter: the experimenter talked to the child

4. Non-communicative-Mother: the mother gently shook a rattle

5. Non-communicative-Experimenter: the experimenter gently shook a rattle

The order of the conditions (with the exception of the Baseline condition, which was always recorded first) was counterbalanced using a balanced Latin square design. The distance of the mother's or the experimenter's face as well as the distance of the rattle was always at about 20 cm from the infants' face. In order to avoid tactile stimulation, we asked the mothers not to touch their infants during the observation. This way we made sure that the stimulation remained auditory and visual for all conditions. If the infant grew fussy during the observation, the experimenter interrupted the observation and asked the mother to pick him or her up. She waited until the infant regained a state of calm rest and then asked the mother to place the infant back into the baby seat. The observation was then continued with the recording made from exactly the same angle as before interruption. If the infant did not regain a state of calm rest within 15 minutes $(\mathrm{N}=4)$, we made another appointment with the mother and (for the same or the next day) to make the recordings.

\subsection{Measures and quantification of hand movements for analysis}

We quantified hand activity (Hand activity) by measuring the distance travelled by the LH and the $\mathrm{RH}$ on the two-dimensional plane of the video display. Kinovea Version 0.8.15 (available for free download at http://www.kinovea.org) was used to display each video recording frame by frame (10 frames per second) and to place within each frame a marking cursor on the infants' hands, which could then be tracked by the software in order to calculate the distances travelled (in pixels) by each hand. The coder made sure that the cursor always remained in the same location on the infant's body.

We measured duration of self-touch (Self-touch) by the amount of time (in seconds) during which the hands were in contact with the infant's own body. Kinovea Version 0.8.15 was also used to code frame by frame the amount of time during which the LH or the RH was in contact with the head, the mouth, the upper body or the lower body. Figure 1 shows a screenshot of Kinovea in use for coding an infant's limb movements. 
Two independent coders analyzed the trials. We calculated inter-rater reliability for $20 \%$ of the trials (by randomly selecting $20 \%$ of the recordings from each condition) using the intra-class correlation coefficient and found that agreement between the two coders was high, for both Hand activity $(r I C C=0.83)$ and Self-touch measures $(r I C C=0.80)$.

To check for any effect of fatigue from one condition to the other, we compared Hand activity in the second, third, fourth and fifth recording, but found no significant difference $(\mathrm{F}(3,16)=0.95, \mathrm{p}=.42)$.

Differences in Hand activity and Self-touch were each examined with $5 \times 2 \times 2$ (Condition [CommMother, NonCommMother, CommExp, NonCommExp, Basel] $\times$ Age $[2 \mathrm{w}$ vs $3 \mathrm{~m}] \times$ Observed hand [LH vs $\mathrm{RH}]$ ) generalized estimating equations (GEE) analyses. We used Bonferroni procedures to adjust for subsequent multiple comparisons.

\section{Results}

\subsection{Hand activity}

The GEE analysis of Hand activity revealed significant main effect of Condition ( Wald $\chi^{2}=$ 60.71, $d f=4, p=.001)$ as well as a significant Condition $\times$ Hand $\times$ Age interaction $\left(\right.$ Wald $^{2}=$ $332, d f=14, p=.001$, see Figures $2 \mathrm{a}$ and $2 \mathrm{~b}$ ). This suggests that Hand activity depended on the communicative context, that this effect differed for the LH and the RH and was also agedependent. We first present the results of subsequent pairwise comparisons within each age group, followed by comparisons between the two ages, with the significance threshold set at .05 .

\subsubsection{Hand activity at two weeks}

First, we compared Hand activity within each condition. In the Baseline condition, there was no difference between the activities of the LH and the RH, $p=.61$. The only significant difference between the activities of the two hands appeared in one of the two communicative conditions, namely the Communicative-Experimenter condition, where the LH moved significantly more than the $\mathrm{RH}, p=.006$. We found no significant differences between the activities of the two hands within the other conditions (see Table 1a).

Second, we looked at how the activity of each hand varied across experimental conditions at two weeks. Pairwise comparisons showed that the LH moved significantly more in the Communicative-Experimenter condition than in any of the other conditions: compared to the Baseline condition, $p=.03$; compared to the Communicative-Mother condition, $p=$ .001 ; compared to the Non-communicative-Experimenter condition, $p=.03$ and compared to the Non-communicative-Mother condition, $p=.001$. Other pairwise comparisons did not yield further significant differences. 
Finally, we found that infants showed a general tendency to move their hands more in the communicative conditions than in the non-communicative ones ( $\mathrm{LH}$ and $\mathrm{RH}$ tended to move more in the Communicative-Mother than the Non-communicative-Mother condition, $p$ $=.06[\mathrm{LH}]$ and $p=.07[\mathrm{RH}]$; LH moved significantly more in the CommunicativeExperimenter than the Non-communicative-Experimenter condition, $p=.03$ [this was not true for the $\mathrm{RH}$ where the difference was far from significant, $p=.79]$ ).

We can conclude that at two weeks, infants' LH and RH moved about equally within each condition, with one exception. When the experimenter was talking to them, infants moved their LH more than the RH (but not when it was the mother). When the experimenter was talking, the LH was also more active than in any of the other conditions. We also found that both hands tended to move more when the mother or the experimenter was talking to the infants than when they were shaking a rattle. In other words, infants showed a general tendency to produce more hand activity in the communicative situations at this age.

\subsubsection{Hand activity at three months}

Again, we first compared Hand activity within each condition. In the Baseline condition, just like in two-week-olds, there was no difference between the activities of the $\mathrm{LH}$ and the $\mathrm{RH}, p$ $=.69$ ). The only significant difference between the activities of the two hands appeared in one of the two non-communicative conditions, namely the Non-communicative-Experimenter condition, where the $\mathrm{RH}$ was more active than the $\mathrm{LH}, p=.02$ (see Table 1b).

Second, we looked at how the activity of each hand varied across conditions at three months. Pairwise comparisons showed that both hands moved significantly more in the Communicative-Mother condition than in any of the other conditions: compared to the Baseline condition: LH, $p=.001$; $\mathrm{RH}, p=.001$; compared to the CommunicativeExperimenter condition: $\mathrm{LH}, p=.05$; $\mathrm{RH}, p=.05$; compared to the Non-communicativeMother condition: LH, $p=.001$; RH, $p=.001$ and compared to the Non-communicativeExperimenter condition: LH, $p=.001 ; \mathrm{RH}, p=.03$. In the Communicative-Experimenter condition both hands moved more than in the Baseline condition, $p=.01(\mathrm{LH})$ and $p=.01$ (RH) and the LH (but not the RH) moved more than in the Non-communicative-Experimenter condition, $p=.02$. Other pairwise comparisons did not yield significant differences in Hand activity. Thus, no effect was found when comparing the Baseline condition with the Noncommunicative conditions (see Figure 2b).

We can conclude that at three months, asymmetry in hand activity appeared only in the condition where the experimenter was shaking a rattle in front of the infant, with the RH gesturing more than the LH. This may be a preliminary reaching movement towards the novel 
object, the rattle. We also found that at this age, infants reliably produced more hand activity in the Communicative conditions than in the Non-communicative conditions, especially when communicating with the mother.

\subsubsection{Comparison of Hand activity at two weeks and at three months of age}

Finally, we compared the activity of each hand in each condition at two weeks to the activity of the same hand in the same condition at three months of age. Regarding baseline activity, infants' hands moved less as they grew older. This decrease was significant for the LH, $p$ $=.04$, and almost significant for the RH, $p=.07$.

Regarding the Communicative-Mother condition infants moved both their hands significantly more at three months than at two weeks of age: $\mathrm{LH}, p=.01 ; \mathrm{RH}, p=.04$. We found the opposite pattern for the Communicative-Experimenter condition, for which the activity of both hands decreased with age, significantly for the LH, $p=.02$, but not for the RH, $p=.48$. Thus, the asymmetry observed at two weeks when the experimenter was talking to the infants (more LH movements) was no longer observed at three months.

We found no significant change in Hand activity between two weeks and three months for the Non-communicative conditions. However, it is worth noting than the asymmetry in favor of the RH in the Non-communicative-Experimenter condition emerges at three months.

Thus, comparing the two age groups, we can conclude that the change in infants' hand activity from one age to the other was different in the two communicative conditions, with an increase in activity with age towards the mother and a decrease towards the experimenter (as well as in the Baseline condition). In addition, the asymmetry in hand movements changed with age, in favor of the LH in the Communicative-Experimenter condition at two weeks, and in favor of the RH in the Non-communicative-Experimenter condition at three months.

\subsection{Self-touch}

The GEE analysis of Self-touch revealed a significant main effect of Age ( Wald $^{2}=19.62, d f$ $=1, p=.001)$ as well as a significant Condition $\times$ Hand $\times$ Age interaction $\left(\right.$ Wald $^{2}=91.35, d f$ $=13, p=.001$, see Figures $3 \mathrm{a}$ and $3 \mathrm{~b}$ ). This suggests that Self-touch depended on age, that this effect differed for the $\mathrm{LH}$ and the $\mathrm{RH}$ and was also dependent on the communicative context. We first present the results of subsequent pairwise comparisons within each age group, followed by comparisons between the two ages, with the significance threshold set at .05 .

\subsubsection{Self-touch at two weeks}

First, we compared Self-touch with the two hands within each condition. In the Baseline condition, there was no difference between $\mathrm{LH}$ and $\mathrm{RH}, p=.60$. The only significant 
difference between the two hands appeared in Non-communicative-Mother condition, where Self-touch with the RH was significantly longer than with the LH, $p=.01$ (see Table 2a). We found no significant differences in Self-touch between the two hands within the other conditions.

Second, we looked at how Self-touch with the LH and the RH varied across conditions at two weeks (see Figure 3a). Pairwise comparisons showed significant differences in Self-touch with the LH, which was significantly shorter in the Non-communicative-Mother condition than in the other conditions (except for the Baseline condition): compared to the Communicative-Mother condition, $p=.05$; compared to the Non-CommunicativeExperimenter condition, $p=.01$ and compared to the Communicative-Experimenter condition, $p=.001$. We found no significant difference in Self-touch with the RH across experimental conditions.

We can conclude that at two weeks, duration of self-touch was about equal with the LH and the RH within each condition, with one exception. When the mother was shaking a rattle, infants touched their bodies for longer time with the RH than the LH. In this condition, the LH was held away from the body and more so than in any of the other conditions.

\subsubsection{Self-touch at three months}

First, we compared Self-touch with the two hands within each condition. In the Baseline condition, just like at two weeks, there was no difference between LH and RH, $p=.80$. Significant difference between two hands emerged in two conditions. Self-touch with the LH was significantly longer than with the $\mathrm{RH}$ in both the Communicative-Mother condition, $p=$ .01 and in the Non-communicative-Experimenter condition, $p=.02$ (see Table $2 b$ ). We found no significant differences in Self-touch between the two hands within the other conditions.

Second, we looked at how Self-touch with the LH and the RH varied across experimental conditions at three months. Pairwise comparisons showed significant differences in Self-touch with the RH, which was significantly shorter in the Communicative-Mother condition than in the Non-communicative-Mother condition, $p=.02$ and in the Baseline condition, $p=.02$. Self-touch with the RH was also significantly shorter in the Noncommunicative-Experimenter condition than in the Baseline condition, $p=.01$ (see Figure 3b). We found no significant difference in Self-touch with the $\mathrm{RH}$ across experimental conditions.

We can conclude that at three months, duration of self-touch followed specific patterns when the mother was talking to the infant and when the experimenter was shaking a rattle in front of them. In these two conditions, the RH was held away from the body significantly 
more than the LH.

\subsubsection{Comparison of Self-touch at two weeks and at three months of age}

We compared Self-touch with each hand in each condition at two weeks to Self-touch with the same hand in the same condition at three months of age. Self-touch increased for both hands across all conditions as infants grew older. This increase reached significance for both hands in the Baseline condition: $\mathrm{LH}, p=.001$; RH, $p=.001$, and in the CommunicativeExperimenter condition: $\mathrm{LH}, p<.001 ; \mathrm{RH}, p=.007$. In the remaining three conditions, the increase with age was significant for the LH only: Communicative-Mother condition, $p=.005$; Non-communicative-Mother condition, $p=.001$; Non-communicative-Experimenter condition, $p=.015$.

Thus, comparing the two age groups, we can conclude that as they grew older, infants touched their bodies significantly more with the LH in all conditions and with both hands at baseline and in the Communicative-Experimenter condition.

\section{Discussion}

With our present study we wished to explore early asymmetries in infants' hand activity and duration of self-touch across communicative and non-communicative situations, at two weeks and at three months of age. First, we expected that at baseline, when infants are positioned in a symmetrical posture and no stimulation is provided, hand activity and self-touch would not be lateralized at two weeks or at three months of age (Michel \& Harkins, 1986; Rader \& Stern, 1982; DiMercurio et al., 2018). Our data supported this hypothesis, as we found no asymmetries in hand activity or duration of self-touch with each hand in the baseline condition at two weeks or three months of age.

Our second hypothesis was that three-month-old infants would produce different patterns of hand activity and self-touch in the communicative situation with their mother compared to the communicative situation with the stranger. Our results partly supported this hypothesis, as follows.

Regarding hand activity when in communication with the mother, we did not find more $\mathrm{RH}$ than LH movements at three months. A differentiated (but not lateralized) response towards the mother did emerge at this age however, as infants produced more hand activity when she was talking to them than when it was the experimenter or in any other condition. Further differentiation of hand activity towards the mother emerged when we looked at how hand activity changed between two weeks and three months of age. Comparing the two ages, we found that at three months, infants produced more hand activity when their mother was 
talking to them than at two weeks, whereas hand activity towards the experimenter decreased with age.

Regarding hand activity when in communication with a stranger, we expected more LH (vs RH) activity as a response to the novelty of the situation (Trevarthen, 1996). This was only observed at two weeks (and not at three months), when infants produced more LH movements than RH movements in presence of the stranger and more LH movements than towards their mother.

Regarding duration of self-touch at three months, when facing their mother, we expected that infants would produce more self-touch with the LH as a self-regulatory response (Trevarthen, 1996; Grunwald et al., 2014 and Mueller, Martin \& Grunwald, 2019 for selftouch with the $\mathrm{LH}$ in response to cognitive load). Our results confirmed this, as infants touched their body for longer with the $\mathrm{LH}$ than the $\mathrm{RH}$ when their mother was talking to them. Thus, the asymmetry that Trevarthen (1996) observed for self-touching movements when the infants were in communication with their mother was supported by our data at three months. When facing the stranger (vs the mother), we expected infants to produce more selftouch with LH as a potential response to the mild stress involved (Reissland et al., 2015), our data, however, did not support this. Still, self-touch with the LH as a self-regulatory response appears to be distinct from spontaneous self-touch, which is not lateralised in infants (DiMercurio et al., 2018).

The asymmetry that Trevarthen (1996) observed around four weeks of age for handraising movements appeared for hand activity at two weeks in our study, when the infant was facing a stranger. When infants were in communication with their mother, we did not observe asymmetry either at two weeks or at three months for hand activity. This may be due to methodological differences, as Trevarthen compared hand-raising movements and not hand activity. It is difficult to code and quantify hand raising gestures, as clear criteria regarding, for instance the amplitude and frequency of one such movement unit, are missing. We chose to quantify behaviors that can be coded accurately (quantity of hand activity) and hypothesized that, if such asymmetry exists, it should be present for other movement criteria as well, not only hand raising.

Our third hypothesis was that in presence of an object, that is in our non-communicative conditions where a rattle is presented at midline, three-month-old infants would produce prereaching movements, with more $\mathrm{RH}$ than $\mathrm{LH}$ activity directed toward the target (Michel \& Harkins, 1986; Rader \& Stern, 1982, though see Lynch et al., 2008, for different results). Our data supported this hypothesis because at three months, infants produced more hand activity 
with their RH than their LH when the experimenter was shaking a rattle in front of them. Correspondingly, in this same condition, the $\mathrm{RH}$ was held away from the body significantly more than the $\mathrm{LH}$, which may indicate that in this condition infants were preparing to reach for the object. We did not observe such asymmetry when the mother was shaking the rattle, which might reflect the infant's greater social interest toward the mother than toward the experimenter.

We expected a RH preference for hand activity in the communicative situations as well as for non-communicative pre-reaching actions at three months (e.g.: Bates et al., 1986; Blake et al., 1994; Kimura, 1973). Our fourth assumption was that, since the lateralization of hand movements in communicative situations seems to develop independently from object-related handedness (Cochet et al., 2011; Jacquet et al., 2012), different patterns of hand activity and self-touch would emerge when comparing hand movements in communicative- and noncommunicative situations to baseline. Our results confirmed early differentiation of communicative hand movements versus non-communicative ones, not in terms of laterality but in terms of the amount of hand activity produced by the infants: at three months, infants moved their hands more in the communicative conditions than in the non-communicative ones and this difference appeared as a tendency already at two weeks of age.

Interestingly, at two weeks, infants' hand activity and duration of self-touch with each hand were asymmetrical when the mother shook the rattle and when the experimenter was talking to them. We observed longer self-touch with the RH, meaning that the LH was held away from the body longer than the $\mathrm{RH}$, in the first condition and more LH activity in the latter. Both situations carry novelty compared to the other conditions: the sound of the rattle associated with the presence of the mother and the sound of the experimenter's voice. Thus, similarly to Trevarthen's (1996) suggestion, these patterns of activity of the LH may be associated to the fact that infants reacted to the novelty of the two situations.

In summary, infants moved their hands less but produced self-touch for longer durations at three months than at two weeks when no stimulation was provided. An age-related change was observed for the total amount of hand activity only in the communicative conditions, with hand activity increasing with age when the mother was talking to the infant, and decreasing with age when it was the experimenter. Duration of self-touch globally increased with age, whether infants were stimulated or not. Regarding the asymmetries of movements between both hands, the analysis of the total length of hand movements showed no asymmetry in the absence of stimulation at either age. Between-hand asymmetry appeared only when infants were stimulated by the experimenter, with more LH movements when the experimenter was 
talking to them at two weeks and more RH movements when she was shaking a rattle at three months. The analysis of self-touch duration with each hand showed no asymmetry in the absence of stimulation at either age. Between-hand asymmetry again appeared in some stimulation conditions: longer duration of self-touch with the RH when the mother was shaking a rattle at two weeks and longer duration of self-touch with the LH when she was talking or when the experimenter was shaking a rattle at three months.

We can conclude that left-right asymmetries appeared less consistently than suggested by previous literature and did not only depend on the communicative nature of the situation. Instead, hand activity and duration of self-touch patterns seemed to be influenced by several factors. The first was age (at baseline, infants moved less but performed self-touch for longer durations at three months than at two weeks; more movements with age when the mother was talking to the infant and less movements with age when it was the experimenter; shifts in asymmetry patterns from one age to the other). The second was the presence of the mother (longer duration of self-touch with the LH than with the RH at three months when their mother was talking to them and globally more hand movements at three months than at two weeks when their mother was talking, with the opposite pattern when it was the experimenter). The third was the novelty of the situation (more LH movements when the unfamiliar female experimenter was talking to the infants and the LH held away from the body longer than the RH when the mother was shaking a rattle at two weeks). Finally, the fourth factor was the presence of an object, possibly triggering pre-reaching movements (more RH movements and longer duration of self-touch with the LH when the experimenter was shaking a rattle at three months).

Our results partly support previous studies that pointed out an early differentiation of communicative hand movements versus non-communicative ones. However, it is in terms of the amount of global hand activity produced by the infants, rather than in those of the laterality of movement that this differentiation emerged in our study: at three months, infants produced more hand activity in the communicative conditions than in the non-communicative ones and this difference appeared as a tendency already at two weeks of age.

One limitation of the present study is that beyond hand activity and duration of self-touch, we did not code the types of hand movements infants made. It is indeed possible, that further asymmetries could be observed if we looked at infants' hand shapes or the directionality (along the vertical or the horizontal axes) of their hand movements across experimental conditions. A second limitation is that we did not have the opportunity to observe the infants' hand gestures beyond the age of three months in order to explore how further asymmetries 
emerge and lead to the later lateralization of communicative and pointing gestures. Thirdly, a few methodological limitations should also be considered when interpreting the results of this study. We analyzed infants' hand movements within a relatively short, 40-second window, because the meticulous frame by frame coding of the sequences did not allow for us to code longer sequences. Infants were placed in baby seats which had linings on the sides, which could have restricted infants' arm movements. We should add however that the infants observed in our study were only a few weeks old, therefore their arms still had ample room to move freely in a standard seat. In laboratory settings it would have been possible to use an apparatus that provides ample neck and trunk support in a sitting position, creating for the infant a state of so-called 'liberated motor activity' (Amiel-Tison \& Grenier, 1983). For the current study, we wished to observe infants in their own environment rather than a laboratory and therefore recorded their hand movements in standard baby seats. Finally, we should note that movement data were obtained by calculating the distances travelled by each limb in the two-dimensional plane of the video display. This method is less precise than threedimensional movement capture systems, as one plane of movement is necessarily lost. Given that the camera filmed the lying infant from directly above, both arms were equally affected by this limitation, therefore our conclusions in terms of lateralization of movement were not biased.

An important perspective at present is to continue longitudinal observations and to follow infants from the first weeks of age up to the end of the first year using the same experimental method and to describe with further precision how hand movements develop and become more and more specific across communicative and non-communicative situations during the first months of life. Correlating infants' vocalizations with their hand movements would also be an important next step to gain more insight about the communicative nature or these movements.

Acknowledgement: the authors gratefully acknowledge the support of the French National Research Agency (ANR, Agence Nationale de la Recherche), grant number ANR-13-BSH20007-01 awarded to Jacqueline Fagard. 


\section{References}

Amiel-Tison, C., \& Grenier, A. (1983). Neurologic Evaluation of the Newborn and the Infant. New York: Masson Publishing.

Bates, E., \& Dick, F. (2002). Language, gesture, and the developing brain. Developmental Psychobiology. https://doi.org/10.1002/dev.10034

Bates, E., O’Connell, B., Vaid, J., Sledge, P., \& Oakes, L. (1986). Language and Hand Preference in Early Development. Developmental Neuropsychology. https://doi.org/10.1080/87565648609540323

Blake, J., O’Rourke, P., \& Borzellino, G. (1994). Form and function in the development of pointing and reaching gestures. Infant Behavior and Development. https://doi.org/10.1016/0163-6383(94)90055-8

Capirci, O., \& Volterra, V. (2008). Gesture and speech The emergence and development of a strong and changing partnership. Gesture (Vol. 8). https://doi.org/10.1075/gest.8.1.04cap

Cochet, H., Centelles, L., Jover, M., Plachta, S., \& Vauclair, J. (2015). Hand preferences in preschool children: Reaching, pointing and symbolic gestures. Laterality. https://doi.org/10.1080/1357650X.2015.1007057

Cochet, H., Jover, M., \& Vauclair, J. (2011). Hand preference for pointing gestures and bimanual manipulation around the vocabulary spurt period. Journal of Experimental Child Psychology. https://doi.org/10.1016/j.jecp.2011.04.009

Cochet, H., \& Vauclair, J. (2010). Pointing gestures produced by toddlers from 15 to 30 months: Different functions, hand shapes and laterality patterns. Infant Behavior and Development. https://doi.org/10.1016/j.infbeh.2010.04.009

Corbetta, D., \& Bojczyk, K. E. (2002). Infants return to two-handed reaching when they are learning to walk. Journal of Motor Behavior. https://doi.org/10.1080/00222890209601933

de Vries, J. I. P., Wimmers, R. H., Ververs, I. A. P., Hopkins, B., Savelsbergh, G. J. P., \& van Geijn, H. P. (2001). Fetal handedness and head position preference: A developmental study. Developmental Psychobiology. https://doi.org/10.1002/dev.1042

Decasper, A. J., \& Fifer, W. P. (1980). Of human bonding: Newborns prefer their mothers' voices. Science. https://doi.org/10.1126/science.7375928

DiMercurio, A., Connell, J. P., Clark, M., \& Corbetta, D. (2018). A Naturalistic Observation of Spontaneous Touches to the Body and Environment in the First 2 Months of 
Life. Frontiers in Psychology, 9, 2613. https://doi.org/10.3389/fpsyg.2018.02613

Esseily, R., Jacquet, A. Y., \& Fagard, J. (2011). Handedness for grasping objects and pointing and the development of language in 14-month-old infants. Laterality. https://doi.org/10.1080/1357650X.2010.499911

Fagard, J. (2013). Early development of hand preference and language lateralization: Are they linked, and if so, how? Developmental Psychobiology. https://doi.org/10.1002/dev.21131

Fagard, J., \& Dahmen, R. (2004). Cultural influences on the development of lateral preferences: A comparison between French and Tunisian children. Laterality. https://doi.org/10.1080/13576500342000167

Fagard, J., Margules, S., Lopez, C., Granjon, L., \& Huet, V. (2017). How should we test infant handedness? Laterality. https://doi.org/10.1080/1357650X.2016.1192186

Grunwald M., Weiss T., Mueller S., Rall L. (2014). EEG changes caused by spontaneous facial self-touch may represent emotion regulating processes and working memory maintenance. Brain Research. 1557: 111-126. pmid:24530432

Hannan, T. E. (1987). A cross-sequential assessment of the occurrences of pointing in 3- to 12-month-old human infants. Infant Behavior and Development. https://doi.org/10.1016/0163-6383(87)90003-8

Hepper, P. G., Shahidullah, S., \& White, R. (1991). Handedness in the human fetus. Neuropsychologia. https://doi.org/10.1016/0028-3932(91)90080-R

Holowka, S., \& Petitto, L. (2002). Left Hemisphere Cerebral Specialization for Babies While Babbling. Science, 297, 1515 - 1515.

Hopkins, B., Lems, W., Janssen, B., \& Butterworth, G. (1987). Postural and motor asymmetries in newlyborns. Hum Neurobiol.

Jacquet, A.-Y., Esseily, R., Rider, D., \& Fagard, J. (2012). Handedness for grasping objects and declarative pointing: a longitudinal study. Developmental Psychobiology. https://doi.org/10.1002/dev.20572

Kimura, D. (1973). Manual activity during speaking- II. Left-handers. Neuropsychologia. https://doi.org/10.1016/0028-3932(73)90064-X

Knecht, S. (2000). Handedness and hemispheric language dominance in healthy humans. Brain. https://doi.org/10.1093/brain/123.12.2512

Kotwica, K. A., Ferre, C. L., \& Michel, G. F. (2008). Relation of stable hand-use preferences to the development of skill for managing multiple objects from 7 to 13 months of age. Developmental Psychobiology. https://doi.org/10.1002/dev.20311

Kurjak, A., Vecek, N., Hafner, T., Bozek, T., Funduk-Kurjak, B., \& Ujevic, B. (2002). 
Prenatal diagnosis: What does four-dimensional ultrasound add? Journal of Perinatal Medicine. https://doi.org/10.1515/JPM.2002.008

Lynch, A., Lee, H. M., Bhat, A., \& Galloway, J. C. (2008). No stable arm preference during the pre-reaching period: A comparison of right and left hand kinematics with and without a toy present. Developmental Psychobiology. https://doi.org/10.1002/dev.20297

Mahmoudzadeh, M., Dehaene-Lambertz, G., Fournier, M., Kongolo, G., Goudjil, S., Dubois, J., ... Wallois, F. (2013). Syllabic discrimination in premature human infants prior to complete formation of cortical layers. Proceedings of the National Academy of Sciences. https://doi.org/10.1073/pnas.1212220110

McCartney, G., \& Hepper, P. (1999). Development of lateralized behaviour in the human fetus from 12 to 27 weeks' gestation. Developmental Medicine and Child Neurology. https://doi.org/10.1111/j.1469-8749.1999.tb00559.x

Michel, G. F., Babik, I., Nelson, E. L., Campbell, J. M., \& Marcinowski, E. C. (2013). How the development of handedness could contribute to the development of language. Developmental Psychobiology. https://doi.org/10.1002/dev.21121

Michel, G. F., Babik, I., Sheu, C. F., \& Campbell, J. M. (2014). Latent classes in the developmental trajectories of infant handedness. Developmental Psychology. https://doi.org/10.1037/a0033312

Michel, G. F., \& Harkins, D. A. (1986). Postural and lateral asymmetries in the ontogeny of handedness during infancy. Developmental Psychobiology. https://doi.org/10.1002/dev.420190310

Michel, G. F., Tyler, A. N., Ferre, C., \& Sheu, C. F. (2006). The manifestation of infant handuse preferences when reaching for objects during the seven- to thirteen-month age period. In Developmental Psychobiology. https://doi.org/10.1002/dev.20161

Mueller SM, Martin S, Grunwald M (2019). Self-touch: Contact durations and point of touch of spontaneous facial self-touches differ depending on cognitive and emotional load. PLoS ONE 14(3): e0213677. https://doi.org/10.1371/journal.pone.0213677

Myowa-Yamakoshi, M., \& Takeshita, H. (2006). Do human fetuses anticipate self-oriented actions? A study by four-dimensional (4D) ultrasonography. Infancy. https://doi.org/10.1207/s15327078in1003_5

Nelson, E. L., Campbell, J. M., \& Michel, G. F. (2013). Unimanual to bimanual: Tracking the development of handedness from 6 to 24 months. Infant Behavior and Development. https://doi.org/10.1016/j.infbeh.2013.01.009

Rader, N., \& Stern, J. D. (1982). Visually elicited reaching in neonates. Child Development. 
https://doi.org/10.1111/j.1467-8624.1982.tb01364.x

Ramsay, D. S. (1980). Onset of unimanual handedness in infants. Infant Behavior and Development. https://doi.org/10.1016/S0163-6383(80)80045-2

Ramsay, D. S. (1984). Onset of duplicated syllable babbling and unimanual handedness in infancy: Evidence for developmental change in hemispheric specialization? Developmental Psychology. https://doi.org/10.1037/0012-1649.20.1.64

Reissland, N., Aydin, E., Francis, B., \& Exley, K. (2015). Laterality of foetal self-touch in relation to maternal stress. Laterality, 20(1), 82-94. https://doi.org/10.1080/1357650X.2014.920339

Trevarthen, C. (1996). Lateral asymmetries in infancy: Implications for the development of the hemispheres. In Neuroscience and Biobehavioral Reviews. https://doi.org/10.1016/0149-7634(95)00070-4

Van Der Meer, A. L. (1997). Keeping the arm in the limelight: Advanced visual control of arm movements in neonates. European Journal of Paediatric Neurology. https://doi.org/10.1016/S1090-3798(97)80040-2

Vauclair, J., \& Cochet, H. (2013). Hand preference for pointing and language development in toddlers. Developmental Psychobiology. https://doi.org/10.1002/dev.21073

Vauclair, J., \& Imbault, J. (2009). Relationship between manual preferences for object manipulation and pointing gestures in infants and toddlers. Developmental Science. https://doi.org/10.1111/j.1467-7687.2009.00850.x

Ververs, I. A. P., de Vries, J. I. P., van Geijn, H. P., \& Hopkins, B. (1994). Prenatal head position from 12-38 weeks. I. Developmental aspects. Early Human Development. https://doi.org/10.1016/0378-3782(94)90157-0

von Hofsten, C. (1982). Eye-hand coordination in the newborn. Developmental Psychology. https://doi.org/10.1037/0012-1649.18.3.450 


\section{Tables}

Table $1 a$ Hand activity at 2 weeks

Table $1 b$ Hand activity at 3 months

\begin{tabular}{|c|c|c|c|c|c|c|c|}
\hline \multirow[b]{2}{*}{ Age } & \multirow[b]{2}{*}{ Condition } & \multicolumn{2}{|c|}{$\begin{array}{c}\text { Hand } \\
\text { activity (pix) }\end{array}$} & \multirow[b]{2}{*}{ Age } & \multirow[b]{2}{*}{ Condition } & \multicolumn{2}{|c|}{$\begin{array}{c}\text { Hand } \\
\text { activity (pix) }\end{array}$} \\
\hline & & LH & RH & & & LH & RH \\
\hline \multirow{5}{*}{$\begin{array}{l}\frac{n}{0} \\
\frac{D}{0} \\
\vdots \\
N\end{array}$} & Baseline & 2902 & 3083 & \multirow{5}{*}{ 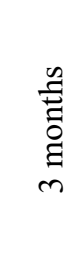 } & Baseline & 1774 & 1856 \\
\hline & Non-communicative-Experimenter & 2708 & 2652 & & Non-communicative-Experimenter & 2019 & 2707 \\
\hline & Communicative-Experimenter & 4108 & 3151 & & Communicative-Experimenter & 2772 & 2835 \\
\hline & Non-communicative-Mother & 1981 & 1888 & & Non-communicative-Mother & 2380 & 2213 \\
\hline & Communicative-Mother & 2364 & 2505 & & Communicative-Mother & 3737 & 3721 \\
\hline
\end{tabular}

We recorded infants' hand movements and quantified hand activity by measuring in pixels the distance travelled by the left hand (LH) and the right hand (RH) on the two-dimensional plane of the video display. Values that are significantly higher for one hand than for the other hand in the same condition are indicated in red and values that are significantly higher for one hand in one condition compared to other conditions are indicated in bold (if a value differ both from the value of the other hand and from the value of the same hand in other conditions, it is indicated in red and bold), see below for the corresponding pairwise comparisons.

Table 1a shows that at two weeks, in the Communicative-Experimenter condition, the LH moved significantly more than the RH, $p=.006$ as well as the same hand in any of the other conditions (Baseline, $p=.03$

Communicative-Mother, $p=.001$; Non-communicative-Experimenter, $p=.03$ and Non-communicative-Mother, $p=.001)$.

Table $1 b$ shows that at three months, in the Non-communicative-Experimenter condition, the RH was more active than the LH, $p=.02$. Also, both hands moved significantly more in the Communicative-Mother condition than in any of the other conditions (Baseline: LH, $p=.001$; $\mathrm{RH}, p=.001$; Communicative-Experimenter: LH, $p$ $=.05$; RH, $p=.05$; Non-communicative-Mother: LH, $p=.001 ; \mathrm{RH}, p=.001$ and Non-communicativeExperimenter: LH, $p=.001 ; \mathrm{RH}, p=.03)$. In the Communicative-Experimenter condition both hands moved more than in the Baseline condition, $p=.01(\mathrm{LH})$ and $p=.01(\mathrm{RH})$. 
Table $2 b$ Self touch at 3 months

\begin{tabular}{|c|c|c|c|c|c|c|c|}
\hline \multirow[b]{2}{*}{ Age } & \multirow[b]{2}{*}{ Condition } & \multicolumn{2}{|c|}{$\begin{array}{l}\text { Self touch } \\
\quad(\mathrm{sec})\end{array}$} & \multirow[b]{2}{*}{ Age } & \multirow[b]{2}{*}{ Condition } & \multicolumn{2}{|c|}{$\begin{array}{l}\text { Self touch } \\
(\mathrm{sec})\end{array}$} \\
\hline & & LH & $\mathrm{RH}$ & & & LH & RH \\
\hline \multirow{5}{*}{$\begin{array}{l}\frac{n}{0} \\
0 \\
0 \\
0 \\
N\end{array}$} & Baseline & 7.95 & 6.86 & \multirow{5}{*}{ 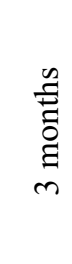 } & Baseline & 24.77 & 24.14 \\
\hline & Non-communicative-Experimenter & 11.30 & 8.98 & & Non-communicative-Experimenter & 22.73 & 15.33 \\
\hline & Communicative-Experimenter & 9.57 & 9.79 & & Communicative-Experimenter & 20.16 & 19.71 \\
\hline & Non-communicative-Mother & 3.12 & 12.41 & & Non-communicative-Mother & 25.40 & 20.77 \\
\hline & Communicative-Mother & 8.97 & 11.73 & & Communicative-Mother & 21.28 & 15.28 \\
\hline
\end{tabular}

We measured self-touch by the amount of time (in seconds) during which the hands were in contact with the infant's own body. Values that differ significantly from others are indicated in red, see below for the corresponding pairwise comparisons.

Table 2a shows that at two weeks, in the Non-communicative-Mother condition, self-touch with the RH was significantly longer than with the LH, $\mathrm{p}=.01$. Self-touch with the LH was significantly shorter in this condition than in the other conditions (except for Baseline): Communicative-Mother, $\mathrm{p}=.05$; Non-CommunicativeExperimenter, $\mathrm{p}=.01$ and Communicative-Experimenter, $\mathrm{p}=.001$.

Table $2 \mathrm{~b}$ shows that at three months, self-touch with the LH was significantly longer than with the RH in both the Communicative-Mother condition, $\mathrm{p}=.01$ and in the Non-communicative-Experimenter condition, $\mathrm{p}=.02$. Also, Self-touch with the RH was significantly shorter in the Communicative-Mother condition than in the Noncommunicative-Mother condition, $\mathrm{p}=.02$ and in the Baseline condition, $\mathrm{p}=.02$. Self-touch with the RH was also significantly shorter in the Non-communicative-Experimenter condition than in the Baseline condition, $p=$ .01 . 


\section{Figures}
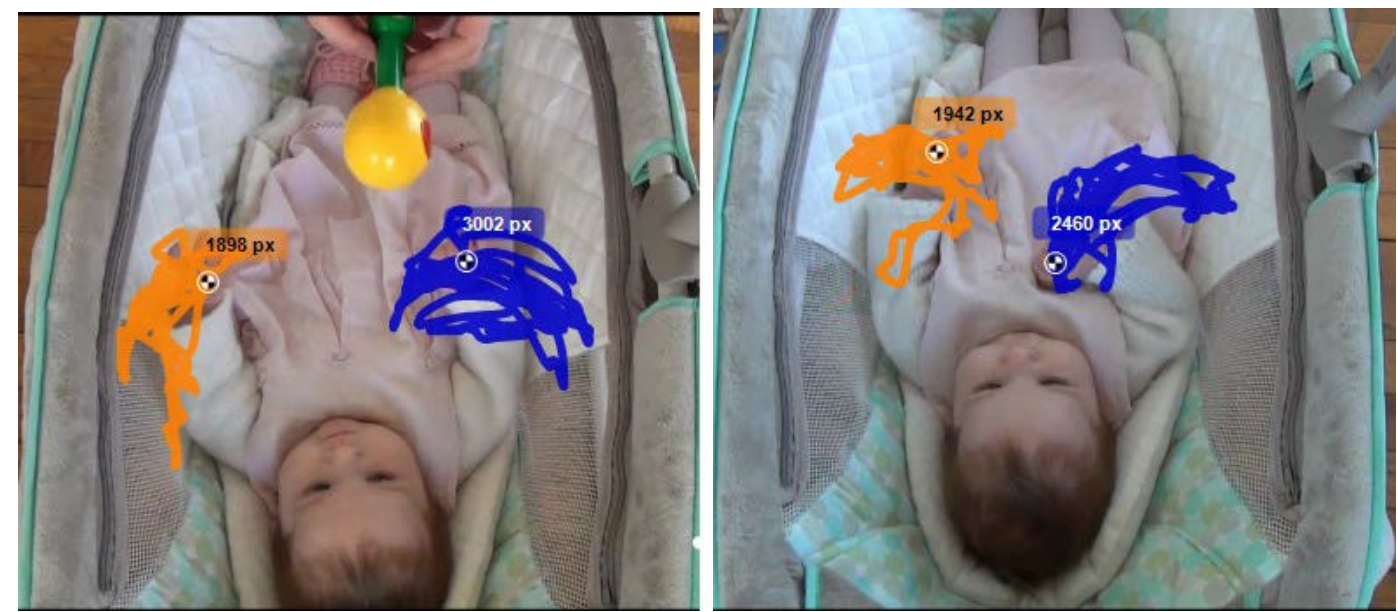

Figure 1 Screenshot of the Kinovea program used for coding an infant's limb movements in a noncommunicative- and a communicative experimental condition. The colored rectangles above each limb indicate the number of pixels travelled by the limb.

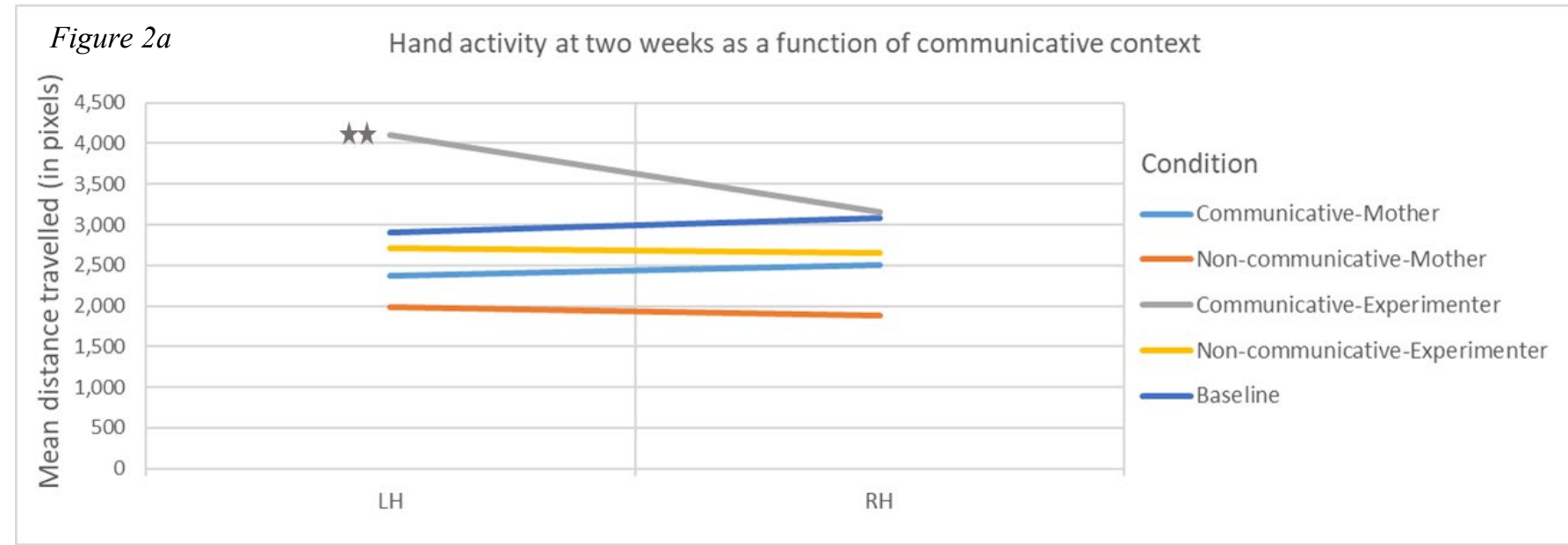

Figure $2 b \quad$ Hand activity at three months as a function of communicative context

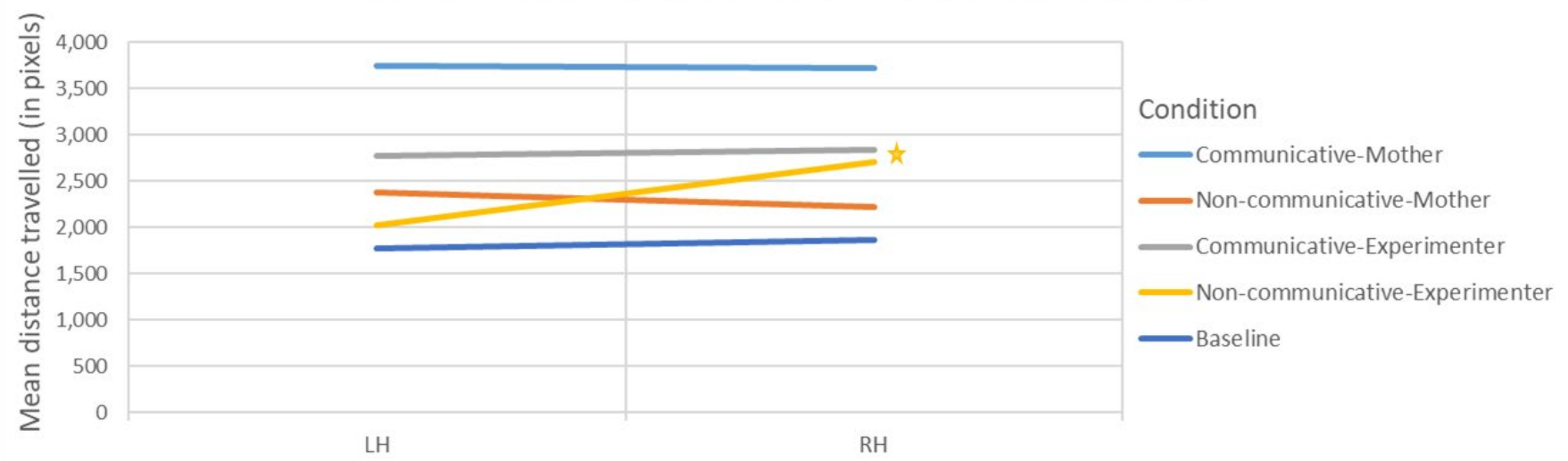

Figures $2 a$ and $2 b$ Two-week-old and three-month old infants' left- and right hand (LH, RH) activity across communicative and non-communicative conditions with either the mother or a female experimenter. Significant differences between means are marked with an asterisk $(* \mathrm{p}<0.05 ; * * \mathrm{p}<0.01)$, as calculated with pairwise comparisons following the Generalized Estimating Equations (GEE) procedure. 


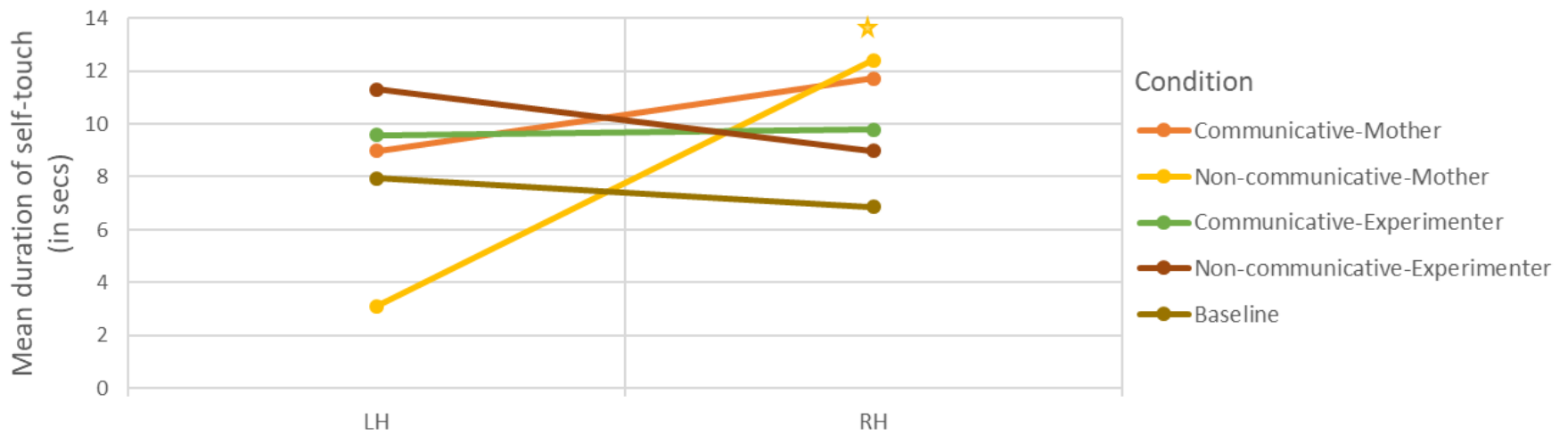

Figure $3 b \quad$ Self-touch at three months as a function of communicative context

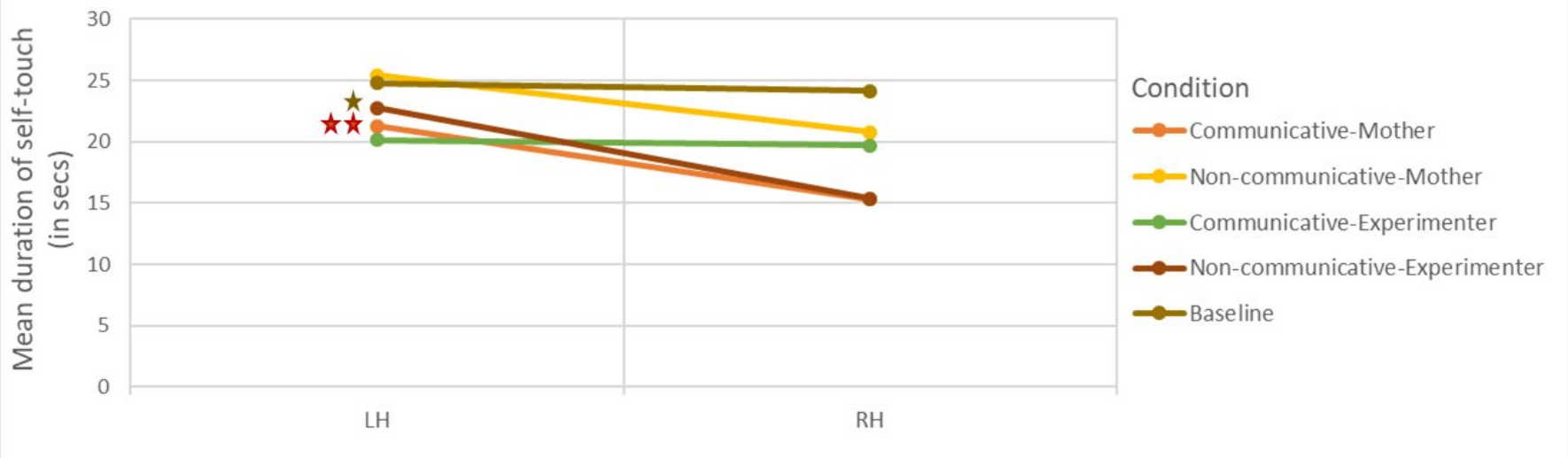

Figure $3 a$ and $3 b$ Mean duration of time two-week-old and three-month old infants spent touching their own body with the left- and right hand ( $\mathrm{LH}, \mathrm{RH})$ across communicative and non-communicative conditions with either the mother or a female experimenter. Significant differences between means are marked with an asterisk $(* \mathrm{p}<0.05 ; * * \mathrm{p}<0.01)$, as calculated with pairwise comparisons following the Generalized Estimating Equations (GEE) procedure. 